\title{
Sofosbuvir May be a Potential Anti-SARS-CoV-2 RdRp Drug
}

\author{
${ }^{1,3}$ Mohamed Nabil Ibrahim and ${ }^{2}$ Abdo Abdellah Elfiky \\ ${ }^{1}$ Department of Clinical Laboratory Sciences, Jouf University, Saudi Arabia \\ ${ }^{2}$ Department of Biophysics, Cairo University, Egypt \\ ${ }^{3}$ Department of Microbiology, Ain Shams University, Egypt
}

\begin{abstract}
Article history
Received: 02-03-2021

Revised: 30-04-2021

Accepted: 03-05-2021

Corresponding Author: Mohamed Nabil Ibrahim Department of Clinical Laboratory Sciences, Jouf University, Saudi Arabia E-mail: mnabil@ju.edu.sa
\end{abstract}

\begin{abstract}
The Coronavirus Diseases 2019 (COVID-19) seriously affecting human health all over the world. More than $107 \mathrm{M}$ people are reported positive for SARS-CoV-2, the virus causing COVID-19 pneumonia, from which $+2.3 \mathrm{M}$ died. Nucleotide Inhibitors (NI) have promising results in terms of its efficacy against different viral polymerases, including the Hepatitis C Virus (HCV) Non-Structural Protein 5 B (NS5B) RNA dependent RNA polymerase (RdRp) 1. Thus, the non-structural protein 12 (nsp12) RdRp of the human coronavirus represents an attractive target to develop a possible therapeutic agent. Sofosbuvir proved itself as a potential anti-SARS-CoV-2 RdRp and could inhibit viral replication and infection propagation.
\end{abstract}

Keywords: Sofosbuvir-COVID-19-RNA Polymerase-Therapy

\section{Dear Editor}

Coronavirus Diseases 2019 (COVID-19) seriously affecting human health all over the world. More than $149 \mathrm{M}$ people are reported positive for SARS-CoV-2, the virus causing COVID-19 pneumonia, from which $+3.15 \mathrm{M}$ died. Nucleotide Inhibitors (NI) have promising results in terms of their efficacy against different viral polymerases, including the Hepatitis C Virus (HCV) Non-Structural Protein 5 B (NS5B) RNA dependent RNA polymerase (RdRp) (Gane et al., 2013). Thus, the non-structural protein 12 (nsp12) RdRp of the human coronavirus represents an attractive target to develop a possible therapeutic agent.

The Ebola virus NI, Remdesivir, was authorized for emergency use by the Food and Drugs Administration (FDA) against COVID-19 in May 2020. Various studies reported the in silico effectiveness of other nucleotide inhibitors currently in the market against SARS-CoV-2 (Elfiky, 2020a). The study utilized a structure of SARSCoV-2 RdRp for small molecule screening. Also, protein dynamics were simulated for 100 ns. Sofosbuvir is currently used for HCV treatment since the FDA approved it in 2013 (Gane et al., 2013). The safety profile of Sofosbuvir was tested over millions of people worldwide and different companies are manufacturing it at affordable prices in low-income countries like India and Egypt (Omar et al., 2018; Elsharkawy et al., 2017; Shah et al., 2017). Sofosbuvir is able to bind to RdRp of SARS-CoV-2 with comparable binding energies compared to Remdesivir (Fig. 1) (Elfiky, 2020b; Jácome et al., 2020; Elfiky et al., 2021).
The dynamics were simulated using the NAMD software utilizing the CHARMM36 force field (Huang and MacKerell, 2013; Phillips et al., 2005). A water box (TIP3P model) is added to the system with added $\mathrm{NaCl}$ salt concentration adjusted to be in the physiological value (154 mM) (Mark and Nilsson, 2001). After dynamics, Maestro is utilized to cluster the trajectories to be tested using the docking protocol (Schrödinger, 2017-1). AutoDock Vina is used in the docking study, while AutoDock tools and PyMOL are used to prepare the docking files and to analyze the data (Rauf et al., 2015; Trott and Olson, 2010). The docking grid was adjusted to cover the active site aspartates (D760 and D761) using the flexible ligand and flexible active site protocol.

As reflected in Fig. 1, the Sofosbuvir is successfully fitted inside the nucleotide channel by forming contacts with the channel-lining residues (blue sticks in the lower enlarged panel of Fig. 1). Six H-bonds (with S549, R555(2), R624(2) and N691), four hydrophobic contact (with Y455, K551, R553 and K621) and two salt bridges (with R555 and R624) are established.

The binding affinity of Sofosbuvir $(-7.4 \pm 0.3 \mathrm{kcal} / \mathrm{mol})$ is very close to that for Remdesivir $(-7.3 \pm 0.1 \mathrm{kcal} / \mathrm{mol})$ against SARS-CoV-2 RdRp. This affinity is persistent during $25 \mathrm{~ns}$ MDS run on the formed complex. In previous studies on a model for SARS-CoV-2 RdRp built before the first structure was released, Sofosbuvir shows similar results regarding the binding affinity and the binding mode (Elfiky, 2020a-c; Jácome et al., 2020). Sofosbuvir proved its safety and anti-HCV activity during the last eight years and may be a potential SARS-CoV-2 inhibitor. 


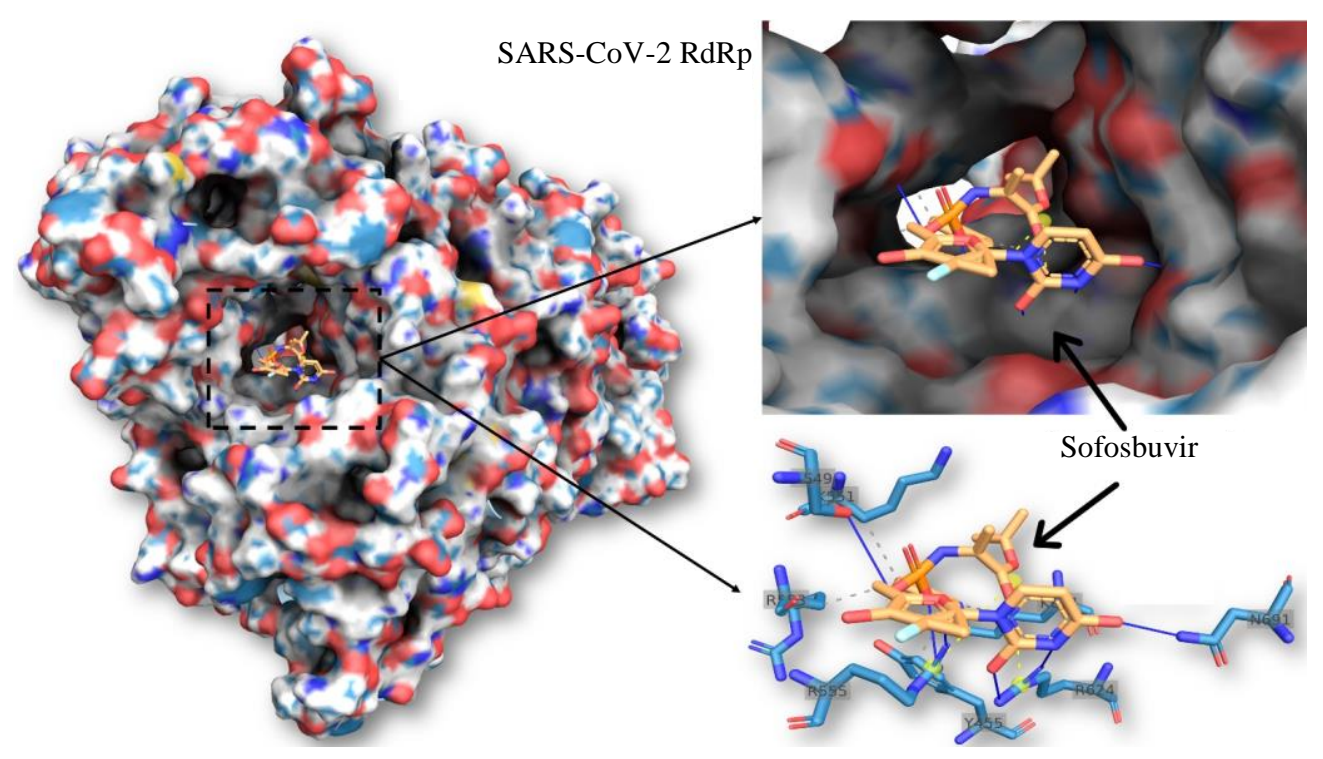

Fig. 1: Sofosbuvir at SARS-COV-2 RdRp active site pocket. The docking pose of sofosbuvir (orange sticks) in the active site of SARSCOV-2 RdRp (conformation at $28.1 \mathrm{~ns}$ ) (surface) depicted by PyMOL software. The interactions are shown in blue, dashed gray and dashed-yellow lines for H-bonding, hydrophobic contacts and salt bridges. The SARS-COV-2 RdRp interacting residues are shown in blue stick in the lower enlarged panel and labeled by its one-letter code

Sofosbuvir shows a promaount binding affinity to the RdRp and tightly interacting with the binding site; hence, supposed to be the right candidate against COVID-19. It could be used to inhibit SARS-CoV-2 RdRp, which yet to be confirmed through experimental binding assays. Currently, we are working on modified sofosbuvir compounds to optimize the drug for SARS-CoV-2 RdRp further. Conclusively, in vitro binding studies and in vivo studies needed to confirm our suggestion that Sofosbuvir could be a therapeutic option against SARS-CoV-2 by targeting its polymerase.

\section{Acknowledgement}

Computational support from the King Abdullah University of Science and Technology (KAUST HPC allocation grant "K1482") and COVID-19 Grant by Cairo University, is acknowledged.

\section{Ethics}

This article is original and contains unpublished material. The corresponding author confirms that all of the other authors have read and approved the manuscript and no ethical issues involved.

\section{Authors Contributions}

Mohamed Nabil: Funding, revisions and final form of the manuscript.

Abdo A Elfiky: Drafting the manuscript and figure Generation.

\section{Reference}

Elfiky, A. A. (2020a). Anti-HCV, nucleotide inhibitors, repurposing against COVID-19. Life Sciences, 248 ,

117477. https://doi.org/10.1016/j.lfs.2020.117477

Elfiky, A. A. (2020b). Ribavirin, Remdesivir, Sofosbuvir, Galidesivir and Tenofovir against SARS-CoV-2 RNA dependent RNA polymerase (RdRp): A molecular docking study. Life Sciences, 253 ,

117592. https://doi.org/10.1016/j.lfs.2020.117592

Elfiky, A. A. (2020c). SARS-CoV-2 RNA dependent RNA polymerase ( $\mathrm{RdRp}$ ) targeting: An in silico perspective. Journal of Biomolecular Structure and Dynamics, $1-9$. https://www.tandfonline.com/doi/full/10.1080/07 391102.2020.1761882

Elfiky, A. A., Azzam, E. B., \& Shafaa, M. W. (2021). The anti-HCV, Sofosbuvir, versus the anti-EBOV Remdesivir against SARS-CoV-2 RNA dependent RNA polymerase in silico. Molecular Diversity, 1-11. https://doi.org/10.1007/s11030-020-10178-z

Elsharkawy, A., Fouad, R., El Akel, W., El Raziky, M., Hassany, M., Shiha, G., ... \& Esmat, G. (2017). Sofosbuvir-based treatment regimens: real life results of 14409 chronic HCV genotype 4 patients in Egypt. Alimentary Pharmacology \& Therapeutics, 45(5), 681-687. 
Gane, E. J., Stedman, C. A., Hyland, R. H., Ding, X., Svarovskaia, E., Symonds, W. T., ... \& Berrey, M. M. (2013). Nucleotide polymerase inhibitor sofosbuvir plus ribavirin for hepatitis C. New England Journal of Medicine, 368(1), 34-44. https://www.nejm.org/doi/full/10.1056/nejmoa12 08953

Huang, J., \& MacKerell, Jr, A. D. (2013). CHARMM36 all-atom additive protein force field: Validation based on comparison to NMR data. Journal of Computational Chemistry, 34(25), 2135-2145. https://doi.org/10.1002/jcc. 23354

Jácome, R., Campillo-Balderas, J. A., de León, S. P., Becerra, A., \& Lazcano, A. (2020). Sofosbuvir as a potential alternative to treat the SARS-CoV-2 epidemic. Scientific Reports, 10(1), 1-5. https:/www.nature.com/articles/s41598-020-66440-9

Mark, P., \& Nilsson, L. (2001). Structure and dynamics of the TIP3P, SPC and SPC/E water models at $298 \mathrm{~K}$. The Journal of Physical Chemistry A, 105(43), 9954-9960. https://pubs.acs.org/doi/abs/10.1021/jp003020w

Omar, H., El Akel, W., Elbaz, T., El Kassas, M., Elsaeed, K., El Shazly, H., ... \& El Shazly, Y. (2018). Generic daclatasvir plus sofosbuvir, with or without ribavirin, in treatment of chronic hepatitis $\mathrm{C}$ : real-world results from 18378 patients in Egypt. Alimentary pharmacology \& therapeutics, 47(3), 421-431.
Phillips, J. C., Braun, R., Wang, W., Gumbart, J., Tajkhorshid, E., Villa, E., ... \& Schulten, K. (2005). Scalable molecular dynamics with NAMD. Journal of Computational Chemistry, 26(16), 1781-1802. https://doi.org/10.1002/jcc.20289

Rauf, M. A., Zubair, S., \& Azhar, A. (2015). Ligand docking and binding site analysis with pymol and autodock/vina. International Journal of Basic and Applied Sciences, 4(2), 168.

Schrödinger, R. (2017-1). Maestro, Schrödinger, LLC, New York, NY, 2017. https://doi.org/10.1111/jvh.12654

Shah, S., Shah, S. R., Chowdhury, A., Mehta, R., Kapoor, D., Duseja, A., Koshy, A., ... \& Sarin, S. (2017). Sofosbuvir plus ribavirin in treatment-naïve patients with chronic hepatitis $\mathrm{C}$ virus genotype 1 or 3 infection in India. Journal of Viral Hepatitis, 24(5), 371-379. https://doi.org/10.1111/jvh.12654

Trott, O., \& Olson, A. J. (2010). Auto Dock Vina: improving the speed and accuracy of docking with a new scoring function, efficient optimization and multithreading. Journal of Computational Chemistry, 31(2), 455-461. 